\title{
Tritylamine as an ammonia synthetic equivalent: preparation of primary amides
}

\author{
Vassiliki Theodorou, ${ }^{* a}$ Aris Karkatsoulis, ${ }^{a}$ Maria Kinigopoulou, ${ }^{a}$ Valentine Ragoussis, ${ }^{b}$ and \\ Konstantinos Skobridis ${ }^{\mathrm{a}}$ \\ ${ }^{a}$ Department of Chemistry, University of Ioannina, Ioannina, GR-45110, Greece \\ ${ }^{b}$ Department of Chemistry, University of Athens, Panepistimiopolis, Zografou, GR-157 71 \\ Athens, Greece \\ E-mail:vtheodor@cc.uoi.gr
}

\begin{abstract}
A new synthetic route to primary amides via $N$-tritylamides was developed. The initially formed $N$-tritylamides, derived from acylation of tritylamine by activated carboxylic acid derivatives, were deprotected with trifluoroacetic acid at room temperature to the desired primary amides in good yields.
\end{abstract}

Keywords: Tritylamine, tritylamides, ammonia synthetic equivalent, synthesis of primary amides, detritylation

\section{Introduction}

Amide formation is a fundamental reaction of great interest in organic chemistry. The development of efficient methods for the synthesis of amides remains a great challenge because of their importance in chemistry and biology, with a wide range of industrial and pharmaceutical applications and as valuable intermediates in organic synthesis. The classical method for the synthesis of amides is the reaction of amines with carboxylic acids, usually in the presence of coupling reagents, or with carboxylic acid derivatives, acyl halides, anhydrides, esters and other activated species in the presence of a tertiary amine. ${ }^{1}$ The conversion of aldehydes, ketones, oximes $^{2}$ and nitriles, ${ }^{3}$ as well as new reported systems, constitute, also, effective methods to access carboxamides. ${ }^{4-6}$ Traditional methods for the synthesis of primary amides rely on the treatment of acyl halides, acid anhydrides, esters or acids with ammonia. ${ }^{1,7}$ However, the use of ammonia can often be hampered by handling issues, and, additionally, since ammonia can react with carboxylic acids, esters and other functional groups, these methods are sometimes not suitable for selective amide generation. Difficulties can arise also from insolubility of starting 
materials and products, or by competitive hydrolysis of other functional groups by hydroxide anion, when aqueous ammonia is used. On the other hand, the use of ammonia, or generally of amines, is associated with other problems, such as epimerization and/or undesirable deblocking of protective groups in peptide synthesis. Modified methods have proposed the use of other ammonia precursors for the conversion to the corresponding amides. ${ }^{7-10}$

Procedures allowing the formation of carboxamides, without undesirable side-products, would be helpful in organic chemistry. In the course of our ongoing interest in tritylamine reactions and its use for the preparation of primary amines, ${ }^{11}$ we have designed the synthesis of primary amides from $N$-tritylamides, derived by aminotritylation of carboxylic acid derivatives with tritylamine, followed by trityl deprotection (Scheme 1). The easily accessible triphenylmethylamine (tritylamine, $\left.\mathrm{TrNH}_{2}\right)^{11 \mathrm{a}}$ could act as an effective ammonia synthon for the one-pot synthesis of $N$-substituted tritylamides, which subsequently could be deprotected with TFA $^{11 a, 12}$ (Schemes 1-6). Alternatively, one could use the mono-, di- or trimethoxy- tritylamine derivatives, which, as expected, show higher nucleophilicity than tritylamine itself, and are more acid labile, allowing milder conditions for their removal. These compounds are viscous gums, not so easy to be handled, while tritylamine is a crystalline compound commercially available and conveniently weighed.

It is well known that trityl moiety (Tr) is a valuable acid-labile bulky $N, O$ and $S$-protective group, used in peptide, carbohydrate and nucleotide chemistry, readily introduced with trityl chloride, ${ }^{13}$ while tritylamine was used as a synthetic equivalent of ammonia for the preparation of novel synthetic trityl intermediates. ${ }^{11,14}$ Trityl group has also been reported to be installed for the protection of primary amides as $\mathrm{N}$-tritylamides, by the use of triphenylcarbinol, in the presence of $\mathrm{Ac}_{2} \mathrm{O}, \mathrm{H}_{2} \mathrm{SO}_{4}$ and $\mathrm{AcOH},{ }^{15}$ which at a later time were deblocked with acid. On the contrary, in this report, trityl group was incorporated in the form of $\mathrm{TrNH}_{2}$ as a means to introduce nitrogen into the molecule, rather than to protect it. To the best of our knowledge, $\mathrm{TrNH}_{2}$ was occasionally used effectively for the formation of the carboxamide group $\left(\mathrm{CONH}_{2}\right){ }^{14 c, d}$ but there is no precedent for its general application in the synthesis of primary amides.

In order to establish a general, simple and effective method for the synthesis of carboxamides, we performed experiments on a range of carboxylic acids and derivatives, which were successfully converted to the corresponding primary amides (Table 1).

\section{Results and Discussion}

Scheme 1 illustrates our synthetic route starting from the appropriate carboxylic acids or derivatives which were converted via their tritylamides into the corresponding primary amides. Esters do not react with $\mathrm{TrNH}_{2}$ under the reaction conditions, besides, $\mathrm{TrNH}_{2}$ is a poor nucleophile. 


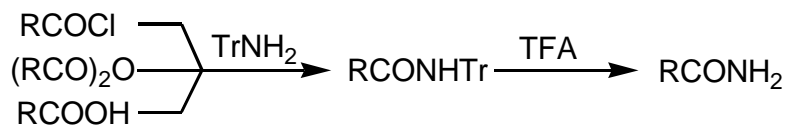

\section{Scheme 1}

Acyl chlorides (entries 1-7, Table 1) reacted readily with $\mathrm{TrNH}_{2}$ giving $N$-tritylamides. The chlorides were prepared from the parent acids immediately before use. Aliphatic and aromatic carboxylic acids were readily converted to the corresponding chlorides with thionyl or oxalyl chloride, in the presence of a tertiary amine, in dichloromethane or toluene as the reaction solvent. After removal of the solvent and chloride excess, and without further purification, acyl chlorides were treated with $\mathrm{TrNH}_{2}$ (Scheme 2).

It should be mentioned that acetylsalicylic acid, aspirin, was readily converted to acetylsalicyloyl chloride with $(\mathrm{COCl})_{2}$, which after reaction with $\mathrm{TrNH}_{2}$ followed by detritylation, gave the expected acetylsalicylamide, without any rearrangement, ${ }^{16}$ as exhibited from both ${ }^{1} \mathrm{H}$ NMR and IR spectra. Moreover, trans-3-nonenoic acid was converted to the expected trans-3-nonenamide, through its acyl chloride, without isomerization, while the tritylamides derived from trans-2-octenoic and trans-2-decenoic acids were completely isomerized to their $\beta, \gamma$-unsaturated derivatives. This isomerization of the $\alpha, \beta$ - to the $\beta, \gamma$ unsaturated compounds takes place in the presence of $\mathrm{Et}_{3} \mathrm{~N}$, but not with pyridine, and is under investigation in our laboratory, to fully understand this behavior.

$$
\mathrm{RCOCl}+\mathrm{TrNH}_{2} \stackrel{\mathrm{Et}_{3} \mathrm{~N}}{\longrightarrow} \mathrm{RCONHTr}
$$

\section{Scheme 2}

Aliphatic anhydrides reacted smoothly with $\mathrm{TrNH}_{2}$ in dichloromethane solution (entries 815, Table 1), with concurrent precipitation of the white crystalline product, almost pure, tritylamide, taken by filtration (Scheme 3). Chain extension and aromaticity increases the reaction time. Cyclic anhydrides reacted with equal ease, as acyclic, to the corresponding $N$-trityl carboxamidocarboxylic acids.

$$
(\mathrm{RCO})_{2} \mathrm{O}+\mathrm{TrNH}_{2} \longrightarrow \mathrm{RCONHTr}+\mathrm{RCOOH}
$$

\section{Scheme 3}

Carboxylic acids (entries 16, 17, Table 1) were effectively coupled (Scheme 4) with the bulky tritylamine by the use of bromotripyrrolidinophosphonium hexafluorophosphate (PyBrOP), a peptide coupling reagent suggested for sterically hindered reactants. ${ }^{14 c, 17}$ The tritylamides derived from $\mathrm{CH}_{3} \mathrm{COOH}$ and $\mathrm{C}_{6} \mathrm{H}_{5} \mathrm{COOH}$ (8a and 11a), are exactly the same with 
tritylamides derived from the corresponding anhydrides (entries 8 and 11 respectively), as expected (see Table 1). Attempts to couple $\mathrm{TrNH}_{2}$ with some protected amino acids (Boc-Pro$\mathrm{OH}$, Ac-Pro-OH and Fmoc-Phe-OH, entries 18-20), in the presence of PyBrOP, were not very successful and moderate yields were attained, due to the formation of byproducts and difficulties in the separation of the products.

$$
\mathrm{RCOOH}+\mathrm{TrNH}_{2} \stackrel{\text { PyBrOP }, \mathrm{Et}_{3} \mathrm{~N}}{\longrightarrow} \mathrm{RCONHTr}
$$

\section{Scheme 4}

$\mathrm{N}$-Detritylation was obtained quantitatively in all the cases by treatment of all tritylamides with $\mathrm{CF}_{3} \mathrm{COOH}$ in $\mathrm{CH}_{2} \mathrm{Cl}_{2}$ (3:1), followed by the addition of triisopropylsilane or methanol (Scheme 5). The trityl group was released as triphenylmethane or triphenylmethylether, respectively, to form the primary amide $\mathrm{RCONH}_{2}$. In the case of Boc-Pro-NHTr, the Bocprotective group was immediately removed by the acid, almost in 5 minutes at rt, followed by the removal of the trityl group ( $\sim 1 \mathrm{~h}$ at $\mathrm{rt})$. Importantly, susceptible functional groups, such as esters, double bonds and the base labile group Fmoc, remain intact under the reaction conditions, while the L-amino acid derivatives retain their configuration.

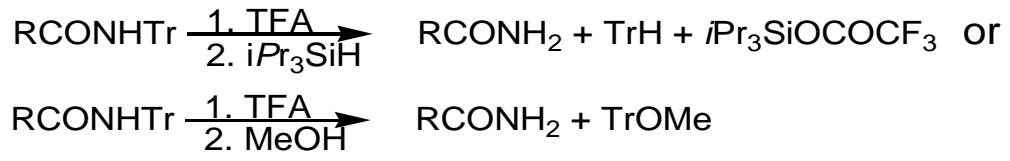

\section{Scheme 5}

All the amides were prepared under mild conditions, which allowed selective aminotritylation/ detritylation of activated carboxyl groups. In general, the reactions and electronic effects in every category of compounds. The isolation procedures were adopted depending on the solubility of amides. In most cases, the products were obtained as pure crystalline compounds, or as a mixture, which could be easily separated by column chromatography on silica gel. The final products are already known compounds and their analytical data are consistent with literature values. Their identity was confirmed by comparison of their spectral and physical properties with those of authentic samples, which are in full agreement. The identity of the intermediates $N$-tritylamides (1-20b) was determined by ${ }^{1} \mathrm{H}$ NMR and IR spectroscopy and elemental analysis. From the results, summarized in Table 1, it is obvious that the proposed method works efficiently in most cases and could constitute an advantageous alternative method for the preparation of primary amides. 
Table 1. Synthesis of $N$-tritylamides (1a-20a) and detritylation products (1b-20b)

\begin{tabular}{|c|c|c|c|c|c|c|}
\hline Entry & Substrate & RCONHTr (a) & $\begin{array}{l}\text { Yield } \\
(\%)^{a}\end{array}$ & $\begin{array}{l}\text { m.p. } \\
\left({ }^{\mathrm{O}} \mathrm{C}\right)\end{array}$ & $\mathrm{RCONH}_{2}(\mathbf{b})^{b}$ & $\begin{array}{l}\text { m.p } \\
\left({ }^{\circ} \mathrm{C}\right)^{c}\end{array}$ \\
\hline 1 & $\mathrm{CH}_{3}\left(\mathrm{CH}_{2}\right)_{14} \mathrm{COCl}$ & $\mathrm{CH}_{3}\left(\mathrm{CH}_{2}\right)_{14} \mathrm{CONHTr}$ & 85 & $128-130$ & $\mathrm{CH}_{3}\left(\mathrm{CH}_{2}\right)_{14} \mathrm{CONH}_{2}$ & $\begin{array}{l}102-103 \\
(104)^{18}\end{array}$ \\
\hline 2 & ${ }_{\mathrm{H}}^{\mathrm{CH}_{2} \mathrm{COCl}}$ & $\mathrm{CH}_{\mathrm{H}}^{\mathrm{CH}_{2} \mathrm{CONHTr}}$ & 65 & $196-197$ & $\begin{array}{l}={ }_{4} \mathrm{CH}_{2} \mathrm{CONH}_{2} \\
{ }_{4} \mathrm{H}\end{array}$ & $\begin{array}{c}68-70 \\
(62-64)^{6 c}\end{array}$ \\
\hline 3 & $\begin{array}{l}-\mathrm{COCl} \\
\mathrm{COOCH}_{3}\end{array}$ & $\begin{array}{l}\mathrm{CONHTr} \\
\mathrm{COOCH}_{3}\end{array}$ & 90 & 205-206 & $\begin{array}{l}\mathrm{CONH}_{2} \\
\mathrm{COOCH}_{3}\end{array}$ & $\begin{array}{c}70-73 \\
(72-74)^{19}\end{array}$ \\
\hline 4 & $\mathrm{COCl}$ & CONHTr & 85 & $223-225$ & $\mathrm{NH}_{2}$ & $\begin{array}{c}320-323 \\
(330-333)^{20}\end{array}$ \\
\hline 5 & & $\begin{array}{l}\mathrm{CONHTr} \\
\mathrm{OCOCH}_{3}\end{array}$ & 65 & 159-160 & 3 & $\begin{array}{c}125-126 \\
(120-124)^{16}\end{array}$ \\
\hline 6 & & $\mathrm{O}_{2}$ & 70 & $196-200$ & & $\begin{array}{c}144-146 \\
(147-148)^{21}\end{array}$ \\
\hline 7 & $\mathrm{O}_{2} \mathrm{~N}^{-}$ & $\mathrm{O}_{2} \mathrm{~N}$ & 72 & 203-205 & $\mathrm{O}_{2} \mathrm{~N}^{-}$ & $\begin{array}{l}150-151 \\
(172)^{22}\end{array}$ \\
\hline 8 & $\left(\mathrm{CH}_{3} \mathrm{CO}\right)_{2} \mathrm{O}$ & $\mathrm{CH}_{3} \mathrm{CONHTr}$ & 92 & 206-208 & $\mathrm{CH}_{3} \mathrm{CONH}_{2}$ & $\begin{array}{l}80-81 \\
(82)^{7 \mathrm{a}}\end{array}$ \\
\hline 9 & $\left(\mathrm{CH}_{3} \mathrm{CH}_{2} \mathrm{CO}\right)_{2} \mathrm{O}$ & $\mathrm{CH}_{3} \mathrm{CH}_{2} \mathrm{CONHTr}$ & 85 & 190-191 & $\mathrm{CH}_{3} \mathrm{CH}_{2} \mathrm{CONH}_{2}$ & $\begin{array}{l}78-79 \\
(79)^{7 a}\end{array}$ \\
\hline 10 & $\left\{\mathrm{CH}_{3}\left(\mathrm{CH}_{2}\right)_{3} \mathrm{CO}\right\}_{2} \mathrm{O}$ & $\mathrm{CH}_{3}\left(\mathrm{CH}_{2}\right)_{3} \mathrm{CONHTr}$ & 80 & $180-181$ & $\mathrm{CH}_{3}\left(\mathrm{CH}_{2}\right)_{3} \mathrm{CONH}_{2}$ & $\begin{array}{l}106(106)^{7 a} \\
(102-104)^{5 b}\end{array}$ \\
\hline 11 & $(\mathrm{PhCO})_{2} \mathrm{O}$ & PhCONHTr & 70 & $168-171$ & $\mathrm{PhCONH}_{2}$ & $\begin{array}{c}128 \\
(130)^{7 a}\end{array}$ \\
\hline 12 & $\begin{array}{c}\mathrm{CO} \\
\mathrm{CO} \\
\mathrm{CO}\end{array}$ & $\begin{array}{l}\mathrm{CONHTr} \\
\mathrm{COOH}\end{array}$ & 83 & $187-189$ & $\begin{array}{l}\mathrm{CONH}_{2} \\
\mathrm{COOH}\end{array}$ & $\begin{array}{l}155-157 \\
(158)^{23}\end{array}$ \\
\hline 13 & $\begin{array}{l}\mathrm{C} \\
\mathrm{C} \\
\mathrm{CO}\end{array}$ & $\begin{array}{l}\mathrm{CONHTr} \\
\mathrm{COOH}\end{array}$ & 86 & 163 & $\begin{array}{l}\mathrm{CONH}_{2} \\
\mathrm{COOH}\end{array}$ & $\begin{array}{c}100-103 \\
(102-104)^{2 b, 24}\end{array}$ \\
\hline 14 & & $\begin{array}{l}\mathrm{CONHTr} \\
\mathrm{COOH}\end{array}$ & 82 & $171-173$ & $\overbrace{\mathrm{COOH}}^{\mathrm{CONH}}$ & $\begin{array}{c}154-156 \quad(158- \\
161)^{d}\end{array}$ \\
\hline 15 & & & 73 & $200-201$ & & $\begin{array}{c}145-147 \\
(147-148)^{25}\end{array}$ \\
\hline 16 & $\mathrm{CH}_{3} \mathrm{COOH}$ & $\mathrm{CH}_{3} \mathrm{CONHTr}{ }^{e}$ & 90 & & $\mathrm{CH}_{3} \mathrm{CONH}_{2}$ & See entry 8 \\
\hline 17 & $\mathrm{PhCOOH}$ & $\mathrm{PhCONHTr}^{e}$ & 70 & & $\mathrm{PhCONH}_{2}$ & See entry 11 \\
\hline
\end{tabular}


Table 1. Continued

\begin{tabular}{|c|c|c|c|c|c|c|}
\hline Entry & Substrate & RCONHTr (a) & $\begin{array}{l}\text { Yield } \\
(\%)^{\mathrm{a}}\end{array}$ & $\begin{array}{l}\text { m.p. } \\
(\mathrm{OC})\end{array}$ & RCONH2 (b)b & $\begin{array}{c}\mathrm{m} \cdot \mathrm{p} \\
(\mathrm{OC}) \mathrm{c}\end{array}$ \\
\hline 18 & Boc-Pro-OH & Boc-Pro-NHTr & 62 & 135 & $\mathrm{H}$-Pro-NH $\mathrm{N}_{2}$ & $\begin{array}{c}94-96 \\
(95-97)^{d}\end{array}$ \\
\hline 19 & Ac-Pro-OH & Ac-Pro-NHTr & 30 & - & Ac-Pro- $\mathrm{NH}_{2}$ & $\begin{array}{c}134-135 \\
(136)^{26}\end{array}$ \\
\hline 20 & Fmoc-Phe-OH & Fmoc-Phe-NHTr & 25 & 203-204 & Fmoc-Phe-NH ${ }_{2}$ & $\begin{array}{c}168-170 \\
(162-163)^{27}\end{array}$ \\
\hline
\end{tabular}

${ }^{a}$ Yields are based on carboxylic acid or anhydride. ${ }^{b}$ Detritylation was quantitative for all the samples. Yield: $>85 \%$. ${ }^{c}$ Literature melting points are given in parentheses. ${ }^{d}$ These values have been derived from the pure products supplier: $[\mathrm{a}]_{\mathrm{D}}^{20}=-104^{\circ}, \mathrm{c} 2, \mathrm{C}_{2} \mathrm{H}_{5} \mathrm{OH}\left(-106^{\circ}\right.$, Aldrich Chemicals). ${ }^{e}$ Tritylamides from entries 16 and 17 are identical with $8 \mathrm{a}$ and $11 \mathrm{a}$, respectively.

In the context of our continuing studies on the extension of the use of tritylamine as ammonia synthon, we wished to access secondary amides from the above carboxylic acid derivatives. The readily obtained $N$-tritylamides could be converted to $N$-substituted tertiary tritylamides by suitable amide deprotonation followed by the addition of the alkylating agent, which, later, could be deprotected to the corresponding $N$-substituted secondary amides (Scheme 6). In this regard we obtained $N$-benzylation of $N$-tritylacetamide and its subsequent detritylation to $\mathrm{N}$ benzylacetamide. Moreover, in a similar manner we tried the formation of $N, N^{\prime}$-dimethylation of the $N, N^{\prime}$-ditritylterephthalamide and the $N, O$-dimethylation of $N$-trityl phthalamic acid. The method should be further investigated in order to be extended as a general protocol for the synthesis of secondary amides.

$$
\text { RCONHTr } \underset{2 . n-B u L i}{2 . R^{\prime} X} \text { RCONR'Tr } \frac{\text { 1. TFA }}{2 . \mathrm{Pr}_{3} \mathrm{SiH}} \text { RCONHR' }+\mathrm{TrH}^{2}+i \mathrm{Pr}_{3} \mathrm{SiOCOCF}_{3}
$$

\section{Scheme 6}

\section{Conclusions}

Herein, we report a simple, mild and efficient method for the conversion of several carboxylic acids and derivatives, acid chlorides and anhydrides, to primary amides in good yields using tritylamine, without undesirable side-products. The obtained pure crystalline trityl protected amides are cleaved with TFA, followed by the addition of triisopropylsilane or methanol, and the trityl cation is removed as triphenylmethane ( $\mathrm{TrH})$ or triphenylmethylether ( $\mathrm{TrOMe})$. The easily separated from the reaction mixture $\mathrm{TrH}$ or $\mathrm{TrOMe}$ can be recovered and reconverted to 
tritylamine. Work continues and we are currently exploring the application of this method, as a general protocol, for the synthesis of secondary amides and sulphonamides.

\section{Experimental Section}

\section{Typical procedure for the preparation of tritylamides}

A. From acyl chlorides (entries 1-7). To a solution of the carboxylic acid $(2 \mathrm{mmol})$ in dry dichloromethane (DCM, $5 \mathrm{~mL}$ ) in a flask equipped with a drying tube, oxalyl chloride $(5 \mathrm{mmol})$ was added at $0{ }^{\circ} \mathrm{C}$ and two drops of DMF. The mixture was allowed to stir for 15 minutes at $0{ }^{\circ} \mathrm{C}$ and for $\sim 2 \mathrm{~h}$ at $\mathrm{rt}$, until no gas was observed. The solvent and excess of oxalyl chloride were then distilled off to dryness. The obtained acyl chloride, without further purification, was dissolved in dry DCM $(10 \mathrm{~mL})$, cooled at $0{ }^{\circ} \mathrm{C}, \mathrm{Et}_{3} \mathrm{~N}(5-10 \mathrm{mmol})$ was added under stirring followed by $\mathrm{TrNH}_{2}(2 \mathrm{mmol})$ in DCM $(5 \mathrm{~mL})$. The resulting mixture was allowed to stir at $\mathrm{rt}$ the appropriate time, until complete conversion, as indicated by TLC. The solvent and excess of oxalyl chloride were then removed, the residue was redissolved in DCM or ethyl acetate (AcOEt), washed with water, a citric acid solution ( $10 \%$ in water) and brine, dried over anhydrous $\mathrm{Na}_{2} \mathrm{SO}_{4}$, concentrated in vacuo and purified by column chromatography on silica gel (methanol/dichloromethane, 1:20-1:10 or ethyl acetate-hexane, 1:20). Yield: $65-90 \%$.

(1a). ${ }^{1} \mathrm{H}$ NMR $\left(250 \mathrm{MHz}, \mathrm{CDCl}_{3}\right): \delta 0.88\left(\mathrm{t}, 3 \mathrm{H}, J=7.00 \mathrm{~Hz}, \mathrm{CH}_{3}\right), 1.25(\mathrm{~m}, 24 \mathrm{H}), 1.58\left(\mathrm{~m}, 2 \mathrm{H}, \mathrm{CH}_{2}\right)$, 2.26 (t, $2 \mathrm{H}, J=7.00 \mathrm{~Hz}, \mathrm{CH}_{2}$ ), 6.57 (s, 1H, NH), 7.20-7.26 (m, 15H, ArH); IR (KBr) 3256, 3053, 3023, 2920, 2851, $1648 \mathrm{~cm}^{-1}$; Anal. Calcd. for $\mathrm{C}_{35} \mathrm{H}_{47} \mathrm{NO}$ : C, 84.45; H, 9.52; N, 2.81; found: C, 84.62; H, 9.47; $\mathrm{N}, 2.71 \%$.

(2a). ${ }^{1} \mathrm{H}$ NMR (250 MHz, $\left.\mathrm{CDCl}_{3}\right): \delta 0.87\left(\mathrm{t}, 3 \mathrm{H}, J=6.75 \mathrm{~Hz}, \mathrm{CH}_{3}\right), 1.20-1.28(\mathrm{~m}, 6 \mathrm{H}), 2.05\left(\mathrm{~m}, 2 \mathrm{H}, \mathrm{CH}_{2}\right)$, $3.00\left(\mathrm{~d}, 2 \mathrm{H}, J=7.00 \mathrm{~Hz}, \mathrm{CH}_{2}\right), 5.65(\mathrm{~m}, 2 \mathrm{H}, \mathrm{CH}=\mathrm{CH}), 6.87(\mathrm{~s}, 1 \mathrm{H}, \mathrm{NH}) 7.15-7.30(\mathrm{~m}, 15 \mathrm{H}, \mathrm{ArH}) ; \mathrm{IR}$ (KBr) $3394,3065,3027,1700 \mathrm{~cm}^{-1}$; Anal. Calcd. for $\mathrm{C}_{28} \mathrm{H}_{31} \mathrm{NO}$ : C, 84.59; H, 7.86; N, 3.52; found: $\mathrm{C}$, $84.68 ; \mathrm{H}, 7.78 ; \mathrm{N}, 3.65 \%$.

(3a). ${ }^{1} \mathrm{H}$ NMR ( $\left.250 \mathrm{MHz}, \mathrm{CDCl}_{3}\right): \delta 2.59\left(\mathrm{~d}, 2 \mathrm{H}, J=5.75 \mathrm{~Hz}, \mathrm{CH}_{2}\right), 2.62\left(\mathrm{~d}, 2 \mathrm{H}, J=5.75 \mathrm{~Hz}, \mathrm{CH}_{2}\right), 3.67$ (s, 3H, $\mathrm{OCH}_{3}$ ), 6.79 (s, 1H, NH), 7.20-7.36 (m, 15H, ArH), 7.85 (s, 4H, ArH); IR (KBr) 3271, 1733, 1654 $\mathrm{cm}^{-1}$; Anal. Calcd. for $\mathrm{C}_{24} \mathrm{H}_{23} \mathrm{NO}_{3}$ : C, 77.19; H, 6.21; N, 3.75; found: C, 77.42; H, 6.35; N, 3.60\%.

(4a). ${ }^{1} \mathrm{H}$ NMR $\left(250 \mathrm{MHz}, \mathrm{CDCl}_{3}\right): \delta 7.20-7.32(\mathrm{~m}, 30 \mathrm{H}, \mathrm{ArH}+2 \mathrm{H}, \mathrm{NH}) 7.85(\mathrm{~s}, 4 \mathrm{H}, \mathrm{ArH}) ; \mathrm{IR}(\mathrm{KBr})$ $3390,3056,1660 \mathrm{~cm}^{-1}$; Anal. Calcd. for $\mathrm{C}_{46} \mathrm{H}_{36} \mathrm{~N}_{2} \mathrm{O}_{2}: \mathrm{C}, 85.16 ; \mathrm{H}, 5.59 ; \mathrm{N}, 4.32$; found: $\mathrm{C}, 85.26 ; \mathrm{H}, 5.45$; $\mathrm{N}, 4.43 \%$.

(5a). ${ }^{1} \mathrm{H}$ NMR $\left(250 \mathrm{MHz}, \mathrm{CDCl}_{3}\right): \delta 1.89$ (s, $\left.3 \mathrm{H}, \mathrm{COCH}_{3}\right), 7.08(\mathrm{~d}, 1 \mathrm{H}, J=7.50 \mathrm{~Hz}, \mathrm{ArH}), 7.20-7.38(\mathrm{~m}$, 16H, ArH), 7.47, (dt, $1 \mathrm{H}, J=8.00,1.75 \mathrm{~Hz}, \mathrm{ArH}), 7.61$ (s, $1 \mathrm{H}, \mathrm{NH}), 7.85$ (d, $1 \mathrm{H}, J=8.00,1.75 \mathrm{~Hz}, \mathrm{ArH}) ; \mathrm{IR}$ (KBr) 3367, 1754, $1684 \mathrm{~cm}^{-1}$; Anal. Calcd. for $\mathrm{C}_{28} \mathrm{H}_{23} \mathrm{NO}_{3}$ : C, 77.79; H, 5.50; N, 3.32; found: C, 77.90; $\mathrm{H}$, $5.65 ; \mathrm{N}, 3.48 \%$.

(6a). ${ }^{1} \mathrm{H}$ NMR $\left(250 \mathrm{MHz}, \mathrm{CDCl}_{3}\right): \delta 7.21(\mathrm{~s}, 1 \mathrm{H}, \mathrm{NH}), 7.22-7.38(\mathrm{~m}, 15 \mathrm{H}, \mathrm{ArH}) 7.45(\mathrm{t}, 1 \mathrm{H}, J=7.75 \mathrm{~Hz}$, ArH), 7.72 (dd, $1 \mathrm{H}, J=7.75,1.50 \mathrm{~Hz}, \mathrm{ArH}), 7.79$ (dd, $1 \mathrm{H}, J=7.75,1.50 \mathrm{~Hz}$, ArH); IR (KBr) 3377, 1674, 
1518, $1350 \mathrm{~cm}^{-1}$; Anal. Calcd. for $\mathrm{C}_{26} \mathrm{H}_{19} \mathrm{ClN}_{2} \mathrm{O}_{3}: \mathrm{C}, 70.51 ; \mathrm{H}, 4.32 ; \mathrm{N}, 6.33$; found: $\mathrm{C}, 70.42 ; \mathrm{H}, 4.43 ; \mathrm{N}$, $6.12 \%$.

(7a). ${ }^{1} \mathrm{H}$ NMR (250 MHz, $\mathrm{CDCl}_{3}$ ): $\delta$ 7.26-7.4 (m, 16H, ArH, NH ), 7.77 (d, 1H, J=8.50 Hz, ArH), 8.15 (dd, $1 \mathrm{H}, J=8.5,1.50 \mathrm{~Hz}, \mathrm{ArH}), 8.29$ (d, $1 \mathrm{H}, J=1.50 \mathrm{~Hz}, \mathrm{ArH})$; IR (KBr) 3385, 1672, 1517, $1352 \mathrm{~cm}^{-1}$; Anal. Calcd. for $\mathrm{C}_{26} \mathrm{H}_{19} \mathrm{ClN}_{2} \mathrm{O}_{3}: \mathrm{C}, 70.51 ; \mathrm{H}, 4.32 ; \mathrm{N}, 6.33$; found: $\mathrm{C}, 70.72 ; \mathrm{H}, 4.35 ; \mathrm{N}, 6.22 \%$.

From anhydrides (entries 8-15). To a stirred solution of the anhydride ${ }^{1 \mathrm{c}}(2 \mathrm{mmol})$ in dry DCM (5-10 mL) $\mathrm{TrNH}_{2}(2 \mathrm{mmol})$ was added and the mixture was allowed to stand at rt overnight until completion of the reaction. In the case of the aromatic anhydrides, the process was carried out at $\sim 60{ }^{\circ} \mathrm{C}$ in toluene. The precipitated crystalline, usually pure, tritylamide or the carboxylic acid trityl monoamide, in the case of cyclic anhydrides, was taken by filtration. If necessary, it was purified by the usual workup. Yield: $70-92 \%$.

(8a). ${ }^{1} \mathrm{H}$ NMR (250 MHz, $\mathrm{CDCl}_{3}$ ): $\delta 2.05$ (s, 3H, $\mathrm{COCH}_{3}$ ), 6.61 (s, 1H, NH), 7.15-7.29 (m, 15H, ArH); IR (KBr) 3243, 3055, 3028, $1655 \mathrm{~cm}^{-1}$; Anal. Calcd. for $\mathrm{C}_{21} \mathrm{H}_{19} \mathrm{NO}: \mathrm{C}, 83.69$; H, 6.35; N, 4.65; found: C, $83.55 ; \mathrm{H}, 6.42 ; \mathrm{N}, 4.59 \%$.

(9a). ${ }^{1} \mathrm{H}$ NMR (250 MHz, DMSO-d $\left.{ }_{6}\right): \delta 0.92$ (t, 3H, $J=7.75 \mathrm{~Hz}, \mathrm{CH}_{3}$ ), 2.24 (q, 2H, $J=7.75 \mathrm{~Hz}$ ), 7.12-7.29 (m, 15H, ArH), 8.46 (s, 1H, NH); IR (KBr) 3256, 3050, 3032, 2973, 2876, $1652 \mathrm{~cm}^{-1}$; Anal. Calcd. for $\mathrm{C}_{22} \mathrm{H}_{21} \mathrm{NO}$ : C, 83.78; H, 6.71; N, 4.44; found: C, 83.60; H, 6.60; N, 4.31\%.

(10a). ${ }^{1} \mathrm{H}$ NMR $\left(250 \mathrm{MHz}, \mathrm{DMSO}-\mathrm{d}_{6}\right): \delta 0.81\left(\mathrm{t}, 3 \mathrm{H}, J=7.75 \mathrm{~Hz}, \mathrm{CH}_{3}\right), 1.18$ (sextet, $2 \mathrm{H}, J=7.75 \mathrm{~Hz}$, $\mathrm{CH}_{2}$ ), 1.39 (quintet, $2 \mathrm{H}, J=7.75 \mathrm{~Hz}, \mathrm{CH}_{3} \mathrm{CH}_{2}$ ), 2.23 (t, $2 \mathrm{H}, J=7.75 \mathrm{~Hz}, \mathrm{CH}_{2}$ ), 7.13-7.28 (m, 15H, ArH), 8.49 (s, 1H, NH); IR (KBr) 3263, 3060, 3024, 2957, 2930, $1651 \mathrm{~cm}^{-1}$; Anal. Calcd. for $\mathrm{C}_{24} \mathrm{H}_{25} \mathrm{NO}$ : 83.93; $\mathrm{H}, 7.34 ; \mathrm{N}, 4.08$; found: 83.75; H, 7.28; N, 3.99\%.

(11a). ${ }^{1} \mathrm{H}$ NMR $\left(250 \mathrm{MHz}, \mathrm{CDCl}_{3}\right): \delta$ 7.10-7.77 (m, 20H, ArH), 6.92 (s, 1H, NH); IR (KBr) 3365, 3052, $1681 \mathrm{~cm}^{-1}$; Anal. Calcd. for $\mathrm{C}_{26} \mathrm{H}_{21} \mathrm{NO}$ : C, 85.92; H, 5.82; N, 3.85; found: C, 85.74; H, 5.78; N, 3.71\%. (12a). ${ }^{1} \mathrm{H}$ NMR (250 MHz, DMSO-d 6 ): $\delta 2.37$ (s, 4H, $\mathrm{CH}_{2}$ ), 7.15-7.29 (m, 15H, ArH), 7.90 (br, 2H, $\mathrm{COOH}, \mathrm{NH})$; IR (KBr) 3430, $2905(\mathrm{w}), 1688 \mathrm{~cm}^{-1}$; Anal. Calcd. for $\mathrm{C}_{23} \mathrm{H}_{21} \mathrm{NO}_{3}: \mathrm{C}, 76.86 ; \mathrm{H}, 5.89 ; \mathrm{N}$, 3.90; found: C, 76.78; H, 5.70; N, 3.99\%.

(13a). ${ }^{1} \mathrm{H}$ NMR (250 MHz, DMSO-d $\mathrm{d}_{6}$ ): $\delta 1.68$ (quintet, $2 \mathrm{H}, \mathrm{CH}_{2}$ ), $2.21\left(\mathrm{t}, 4 \mathrm{H}, \mathrm{CH}_{2} \mathrm{CO}\right.$ ), 7.15-7.28 (m, 15H, ArH), 7.50 (br s, 1H, NH); IR (KBr) 3442, 3260, 3065, 2938, 2850, 1684, $1628 \mathrm{~cm}^{-1}$; Anal. Calcd. for $\mathrm{C}_{24} \mathrm{H}_{23} \mathrm{NO}_{3}$ : C, 77.19; $\mathrm{H}, 6.21 ; \mathrm{N}, 3.75$; found: $\mathrm{C}, 77.41 ; \mathrm{H}, 6.18 ; \mathrm{N}, 3.57 \%$.

(14a). ${ }^{1} \mathrm{H}$ NMR $\left(250 \mathrm{MHz}, \mathrm{DMSO}-\mathrm{d}_{6}\right): \delta 6.20(\mathrm{~d}, 1 \mathrm{H}, J=12.50 \mathrm{~Hz},=\mathrm{CH}), 6.60(\mathrm{~d}, 1 \mathrm{H}, J=12.50 \mathrm{~Hz}$, $=\mathrm{CH}), 7.20-7.26(\mathrm{~m}, 15 \mathrm{H}, \mathrm{ArH}), 9.52(\mathrm{~s}, 1 \mathrm{H}, \mathrm{NH})$; IR (KBr) 3250, 3057, 2861, 1710, $1628 \mathrm{~cm}^{-1}$; Anal.

Calcd. for $\mathrm{C}_{23} \mathrm{H}_{19} \mathrm{NO}_{3}$ : C, 77.29; H, 5.36; N, 3.92; found: C, 77.01; H, 5.57; N, 3.68\%.

(15a). ${ }^{1} \mathrm{H}$ NMR (250 MHz, DMSO-d 6 ): $\delta$ 7.07-7.50 (m, 18H, ArH), 7.74 (d, 1H, J=7.50 Hz, ArH), 9.09 (s, 1H, NH); IR (KBr) 3423, 2968 (w), $1677 \mathrm{~cm}^{-1}$; Anal. Calcd. for $\mathrm{C}_{27} \mathrm{H}_{21} \mathrm{NO}_{3}$ : C, 79.59; H, 5.19; N, 3.44; found: C, 79.48; H, 5.45; N, 3.68\%.

From acids with coupling reagent (entries 16-20). To a solution of the carboxylic acid (2 $\mathrm{mmol})$ in dry DCM (5 mL), $\mathrm{TrNH}_{2}(2 \mathrm{mmol}), \operatorname{PyBrOP}(2 \mathrm{mmol})$ and $\mathrm{Et}_{3} \mathrm{~N}(5 \mathrm{mmol})$ in DCM (2 $\mathrm{mL}$ ) was added and the mixture was allowed to stir at $0{ }^{\circ} \mathrm{C}$ for 5 minutes. Stirring was continued overnight at $\mathrm{rt}$ until completion of the reaction. The reaction mixture was then diluted with AcOEt $(50 \mathrm{~mL})$, treated by the usual workup and the crude product was purified by column 
chromatography on silica gel. Yield: 25-90\%. (16a) and (17a). identical with (8a) and (11a), respectively;

(18a). ${ }^{1} \mathrm{H}$ NMR (250 MHz, $\left.\mathrm{CDCl}_{3}\right):{ }^{1} \mathrm{H} \mathrm{NMR}\left(250 \mathrm{MHz}, \mathrm{CDCl}_{3}\right): \delta 1.42$ (s, 9H, $\left.\mathrm{C}\left(\mathrm{CH}_{3}\right)_{3}\right), 1.85$ (m, 4H), 3.46 (t, 2H), 4.42 (dd, 1H), 7.20-7.28 (m, 15H, ArH), 8.24 (s, 1H, NH); IR (KBr) 3347, 3050, 2873, 1689, $1674 \mathrm{~cm}^{-1}$; Anal. Calcd. for $\mathrm{C}_{29} \mathrm{H}_{32} \mathrm{~N}_{2} \mathrm{O}_{3}$ : C, 76.29; H, 7.06; N, 6.14; found: C, 76.51; H, 7.27; N, 6.29\%. (19a). ${ }^{1} \mathrm{H}$ NMR (250 MHz, $\left.\mathrm{CDCl}_{3}\right): \delta, 2.05(\mathrm{~m}, 4 \mathrm{H}), 2.18\left(\mathrm{~s}, 3 \mathrm{H}, \mathrm{COCH}_{3}\right) 3.52(\mathrm{t}, 2 \mathrm{H}), 4.51(\mathrm{dd}, 1 \mathrm{H})$, 7.15-7.40 (m, 15H, ArH), 7.08 (s, 1H, NH); IR (KBr) 3423, 2968 (w), 1677 $\mathrm{cm}^{-1}$; Anal. Calcd. for $\mathrm{C}_{26} \mathrm{H}_{26} \mathrm{~N}_{2} \mathrm{O}_{2}$ : C, 78.36; H, 6.58; N, 7.03; found: C, 78.48; H, 6.46; N, 6.86\%.

(20a). ${ }^{1} \mathrm{H}$ NMR $\left(250 \mathrm{MHz}, \mathrm{CDCl}_{3}\right): \delta 2.90-3.10\left(\mathrm{t}, 2 \mathrm{H}, \mathrm{C}_{\beta} \mathrm{H}_{2}\right), 4.12(\mathrm{t}, 1 \mathrm{H}, \mathrm{CH}), 4.24\left(\mathrm{t}, 1 \mathrm{H}, \mathrm{C}_{\alpha} \mathrm{H}\right), 4.30-$ 4.42 (br dd, 2H, $\mathrm{CH}_{2}$ ), 5.48 (s, 1H, NHCOO), 7.10-7.82 (m, 28H, ArH), 8.04 (s, 1H, NH); IR (KBr) 3404, 3064, 3031, 2952, 1702 $\mathrm{cm}^{-1}$; Anal. Calcd. for $\mathrm{C}_{43} \mathrm{H}_{36} \mathrm{~N}_{2} \mathrm{O}_{3}: \mathrm{C}, 82.14 ; \mathrm{H}, 5.77 ; \mathrm{N}, 4.46$; found: $\mathrm{C}, 82.38 ; \mathrm{H}$, $5.65 ; \mathrm{N}, 4.67 \%$.

\section{Typical procedure for the detritylation of tritylamides}

To a solution of the tritylamide $(1 \mathrm{mmol})$ in DCM $(2 \mathrm{~mL})$, TFA $(6 \mathrm{~mL})$ was added and the reaction mixture was allowed to stand at $\mathrm{rt}$ for 0.5-3 h, until completion of the reaction, and then triisopropylsilane $(\sim 3 \mathrm{mmol})$ was added. The bright yellow colour of the solution was disappeared in a few minutes and after $30 \mathrm{~min}$. the colourless solution was diluted with $\mathrm{CCl}_{4}$ and evaporated to dryness. The residue was redissolved in diethyl ether $(2 \mathrm{~mL})$ and hexane was added for the precipitation of the deprotected amide, a white amorphous solid, usually pure. If necessary, it was purified by column chromatography, or recrystallized from $\mathrm{MeOH} / \mathrm{Et}_{2} \mathrm{O}$. The resulting $\mathrm{Ph}_{3} \mathrm{CH}(\mathrm{TrH})$ remains soluble in hexane. Yield: $>85 \%$.

\section{References}

1. (a) Benz, G. In Comprehensive Organic Synthesis; Trost, B. M. Fleming, I., Eds.; Pergamon: Oxford, 1991; Vol. 6, pp 381-418. (b) Larock, C. Comprehensive Organic Transformations; 2nd Ed.; Wiley-VCH: New York, 1999; pp 1932-1941. (c) Naik, S.; Bhattacharjya, G.; Talukdar, B.; Patel, B. K. Eur. J. Org. Chem. 2004, 1254. (d) Katritzky, A. R.; He, H. Y.; Suzuki, K. J. Org. Chem. 2000, 65, 8210. (d) Katritzky, A. R.; Cai, C.; Singh, S. K. J. Org. Chem. 2006, 71, 3375.

2. (a) Hyan, D.; Jang, C. D. O. Tetrahedron Lett. 2004, 45, 2285. (b) Gunanathan, C.; BenDavid, Y.; Milstein. D. Science 2007, 317, 790.

3. (a) Fujiwara, H.; Ogasawara, Y.; Yamaguchi, K.; Mizuno, N. Angew. Chem. Int. Ed. 2007, 46, 5202. (b) Shie, J. J.; Fang, J. M. J. Org. Chem. 2003, 68, 1158.

4. (a) Perreux, L.; Loupy, A.; Volatron, F. Tetrahedron, 2002, 58, 2155. (b) Wu, X.; Wannberg, J.; Larhed, M. Tetrahedron 2006, 62, 4665. (c) Sureshbabu, V. V.; Hemantha, H. P. Arkivoc 2008, (ii), 243. 
5. McNulty, J.; Krishnamoorthy, V.; Robertson, A. Tetrahedron Lett. 2008, 49, 6344.

6. (a) Moorthy, J. N.; Singhal, N. J. Org. Chem. 2005, 70, 1926. (b) Kabalka, G. W.; Despande, S. M.; Wadgaonkar, P. P. Synth. Commun. 1990, 20, 1445. (c) Yasushi Imada, Y.; Shibata, O.; Murahashi, S. I. J.Organomet. Chem. 1993, 451, 183.

7. (a) Vogel A. Practical Organic Chemistry; Longman Scientific \& Technical and Wiley: New York, 1989, pp 708-710, 1361. (b) Chen, S. T.; Wu, S. H.; Wang, K. T. Synthesis 1989, 37.

8. (a) Wang, W.; McMurray. J. S. Tetrahedron Lett. 1999, 40, 2501. (b) Veitch, G. E.; Bridgwood, K. L.; Ley, S. V. Org. Lett. 2008, 10, 3623.

9. Chern, C. Y.; Huang, Y. P.; Kan, W. M. Tetrahedron Lett. 2003, 44, 1039.

10. (a) Morera, E.; Ortar, G.; Tetrahedron Lett. 1998, 39, 2835. (b) Ueda, K.; Sato,Y.; Mori, M. J. Am. Chem. Soc. 2000, 122, 10722. (c) Takács, E.; Varga, C.; Skoda-Földes, R.; Kollár, L. Tetrahedron Lett. 2007, 48, 2453.

11. (a) Theodorou, V.; Ragoussis, V.; Strongilos, A.; Zelepos, E.; Eleftheriou, A.; Dimitriou, M. Tetrahedron Lett. 2005, 46, 1357and references cited therein. (b) Theodorou, V.; Skobridis, K. Tetrahedron Lett. 2005, 46, 5021. (c) Theodorou, V.; Skobridis, K.; Karkatsoulis, A. Tetrahedron 2007, 63, 4284.

12. Pearson, D. A.; Blanchette, M.; Baker, M. L.; Guinton, C. A. Tetrahedron Lett. 1989, 30, 2739.

13. (a) Greene, T. W.; Wuts, P. G. M. Protective Groups in Organic Synthesis; Wiley: New York, 1991; pp 366-367. (b) Zervas, L.; Theodoropoulos, D. J. Am. Chem. Soc. 1956, 78, 1359.

14. (a) Soroka, M.; Sygmunt, J. Synthesis 1988, 370. (b) Sharma, S. K.; Songster, M. F.; Colpitts, T. L.; Hegyes, P.; Barany, G.; Castellino, F. J. J. Org. Chem. 1993, 58, 4993. (c) Artuso, F.; Sindona, G.; Athanassopoulos, C.; Stavropoulos, G.; Papaioannou, D. Tetrahedron Lett. 1995, 36, 9309. (d) Zhang, W. J.; Berglund, A.; Kao, J. L. F.; Couty, J. P.; Gershengorn, M. C.; Marshall, G. R. J. Am. Chem. Soc. 2003, 125, 1221. (e) Soroka, M.; Goldenman, W. Arkivoc 2003, (xii), 31.

15. (a) Sieber, P.; Riniker, B. Tetrahedron Lett. 1991, 32, 739. (b) Reddy, D.R.; Iqbal, M. A.; Hudkins, R. L.; Messina-McLaughlin, P. A.; Mallamo, J. P. Tetrahedron Lett. 2002, 43, 8063.

16. Gordon, A. J. Tetrahedron 1967, 23, 863.

17. Coste, J.; Frerot, F.; Jouin, P. J. J. Org. Chem. 1994, 59, 2437.

18. Vandevoorde, S.; Jonsson, K.O.; Fowler, C. J.; Lambert, D. M. J. Med. Chem. 2003, 46, 1440.

19. Puertas, S.; Rebolledo, F.; Gotor, V. Tetrahedron 1995, 51, 1495.

20. (a) Caswell, L. R.; Lanier, J. A.; McAdams, M. A. J. Chem. Eng. Data 1972, 17, 269. (b) L. McMaster, L.; Langreck, F. B. J. Am. Chem. Soc. 1917, 39, 103.

21. Arnone, C.; Consiglio, G.; Frenna, V.; Spinelli, D. J. Org. Chem. 1997, 62, 3093.

22. Grohmann, A. Chem. Ber. 1891, 24, 3808.

23. Flavin, M.; Slaughter, C. Biochemistry 1966, 5, 1340. 
24. Castonguay, R.; Lherbet, C.; Keillor, J. W. Bioorg. Med. Chem. 2002, 10, 4185.

25. Underwood Jr. H. W.; Barker, G. E. J. Am. Chem. Soc. 1930, 52, 4082.

26. Mix, H.; Wilcke, F. W.; Langenbeck, W. Chem. Ber. 1958, 91, 2066.

27. Pozdnev, V. F. Tetrahedron Lett. 1995, 36, 7115. 